\title{
Traffic Control and Traffic Management in a Transportation System with Autonomous Vehicles
}

\author{
Peter Wagner
}

\subsection{Motivation}

This paper aims to quantify the effects of autonomous driving on the traffic management level. This involves developing a model of autonomous driving that makes it possible to use human-controlled and autonomous vehicles with only minor modifications. This is important with regard to defining how the instruments of traffic management need to be developed in the future to enable them to handle autonomous vehicles in the transportation system. Of particular interest in this context is mixed traffic, in which normal and autonomously driving vehicles interact with each other. This will presumably be the normal state of affairs on roads for quite some time even after the introduction of autonomous vehicles; it is, therefore, of great practical significance to gain a good understanding of precisely this situation to predict and prevent any systemic effects that may occur.

Since such vehicles do not yet exist, portions of the following observations must be regarded as an initial appraisal of possible developments presented as a scenario. However, modeling of human drivers is likewise far from complete, so the focus in this paper will be on establishing consistent modeling. The objective of the modeling presented here is to describe, as far as possible, human and autonomous vehicles with the same model, distinguished only by the different parameters used. A good example of this is the distance to the vehicle ahead expressed in terms of the time gap: an autonomous vehicle can achieve times of $0.3 \ldots 0.5 \mathrm{~s}$ [1], whereas vehicles driven by humans are legally required to maintain a distance of at least $0.9 \mathrm{~s}$ (in Germany). The legal recommendation is actually $2.0 \mathrm{~s}$, but this is seldom maintained except when traffic volumes are low. In heavy traffic, the value is often significantly lower; the figure for heavily traveled autobahns that occurs

P. Wagner $(\bowtie)$

Institute of Transportation Systems, German Aerospace Centre (DLR),

12489 Berlin, Germany

e-mail: Peter.wagner@dlr.de 
most often is $1.1 \mathrm{~s}$ (see Fig. 15.3), with an average value of $1.4 \mathrm{~s}$. If drivers complied with the legally stipulated specifications, traffic on many roads would come to a standstill much earlier than is currently the case.

This paper builds on the papers by Friedrich [2] and Pavone [3] in this book. While [2] describes the general effects of autonomous vehicles on the transportation system, this paper addresses the modeling of autonomous and human-driven vehicles as well as the effects of autonomous vehicles on traffic management. Paper [3], by contrast, largely ignores questions of traffic flow and traffic control and focuses primarily on the optimal allocation of supply in relation to demand based on the premise that vehicles can be shared. We can quite rightly conclude at this point that a combination of these approaches, together with a correct description of the share of travelers who would opt for transportation via a robotic "mobility-on-demand" system, allows the best possible appraisal of the potential of autonomous vehicles.

The paper also does not consider effects that would result from a fundamentally different organization of transportation. One example of this would be the EU's CityMobil project, in which such scenarios are discussed and examined in greater detail [4].

This paper will examine how autonomous vehicles affect typical traffic management applications by looking at a few examples which have not been developed in all specifics. These examples, in order of increasing complexity, are the simulation of a single traffic signal system (Sect. 15.4), simulation of an intersection controlled by an adaptive traffic signal system (Sect. 15.5), simulation of a green wave (Sect. 15.6) and the simulation of an entire city (Sect. 15.7).

Some of the questions to be considered here can draw on the effects of the introduction of intelligent speed control (autonomous intelligent speed control-AIC) on traffic flow on highways in particular [5]. There is a great deal of literature on this subject; the dissertation [5] and parts of the book [10] provide a more in-depth overview than is possible in this chapter.

One such AIC scenario is highly similar to Use Case \#1 "Interstate Pilot Using Driver for Extended Availability", which in turn (from a traffic-flow standpoint) is a special variant of Use Case \#3, "Full Automation Using Driver for Extended Availability". This is also the use case that plays the most important role in this chapter, notwithstanding the fact that it is rather irrelevant from the traffic-flow standpoint whether the driver is available or not. The availability of the driver could be important if the impact of failures on traffic flow was being examined, but this topic will not be addressed in this book. This would require detailed statistics regarding how frequently something of this sort occurs and under what circumstances - information which is not available at the current stage of technology of autonomous vehicles. The Use Cases \#2 (Autonomous Valet Parking) and \#4 (Vehicle on Demand) play only a minor role in this chapter, although Use Case \#4 should be treated like Use Case \#3 from a traffic-flow standpoint. Use Case \#2 would be interesting because it has an influence on parking search traffic and thus indirectly on traffic demand and thereby also traffic control, but on the traffic management level it would require a significantly more complex approach than can be achieved here-it would 
require, for example, a precise quantification of the parking search traffic in a city. Even the simulation of the city of Braunschweig described in Sect. 15.7 assumes that vehicles that have reached their destinations always immediately find a parking spot.

\subsection{A Model of Driving}

Models that describe how a human drives a vehicle have been around for a long time [6]. Very many of these models (for an overview see [7-10]) - since 1950 more than 100 models have been described solely for the process of following a vehicle driving aheadcan also without further ado be applied as models for autonomous vehicles, albeit with differing parameters for humans and machines as mentioned in Sect. 15.1. It is thus conceptually quite simple to model mixed traffic and quantify its effects on the transportation system as a whole.

In the following, the focus will be on the process of following a vehicle, which is the most important, but not the only relevant process that determines the development of traffic flow on roads.

Every vehicle is described by its position $x(t)$, which depends on the time $t$ and is defined in relation to some reference (e.g. the beginning of the current section of road), by its velocity $v(t)$ and its acceleration $a(t)$; see also Fig. 15.1. In multi-lane traffic, the lane in which the vehicle is driving - the lateral coordinate, or distance of the vehicle from the edge of the road-comes in as a variable as well. Ideally each vehicle should also be indexed; this is circumvented in the following by describing the vehicle driving ahead with uppercase letters $X(t), V(t), A(t)$. With the additional variables gap $g(t)=X(t)-$ $x(t)-\ell$ and difference in velocity $\Delta v(t)=V(t)-v(t)$ (see also Fig. 15.1), the reaction of the following vehicle can then be defined as the acceleration that the vehicle applies in a particular situation:

$$
a=\frac{\mathrm{d}}{\mathrm{d} t} v=\dot{v}=f(v, g, \Delta v)
$$

This abstract Eq. (15.1) could be abstracted even further; lacking, for example, are models for driver errors and fluctuations as well as the modeling of a reaction time. A corresponding error model is introduced in Sect. 15.3, although reaction time, a notoriously thorny construct, is excluded entirely. While measurement data does very

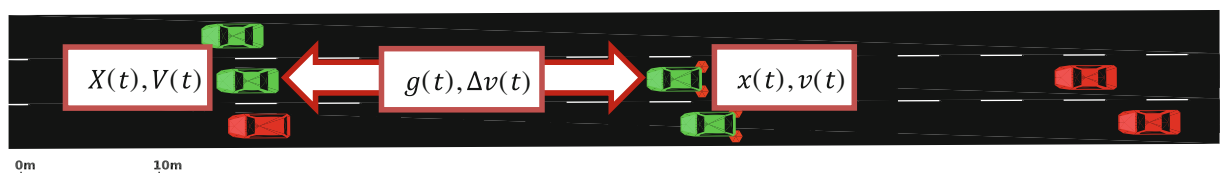

Fig. 15.1 Visualization of the applied dynamic variables using a SUMO [19] screenshot. The traffic direction is from right to left. Image rights: copyright resides with author 
frequently show that the acceleration of a following vehicle lags approximately $2 \mathrm{~s}$ behind the acceleration of the vehicle driving ahead, there are also cases in which the following vehicle starts braking approximately $1 \mathrm{~s}$ before the leading vehicle-for example when approaching a traffic signal (traffic light). In the following, we will examine the abstract Eq. (15.1) with greater specificity. For example, one important question for the following observations is how precisely an autonomous vehicle moves. Surprisingly, many of the current adaptive cruise control systems and also published control algorithms work for automatic vehicles [11-13] as linear control systems:

$$
\dot{v}=\alpha\left(g-g^{*}(v)\right)+\beta \Delta v
$$

Typical parameters for the two time constants are represented by $\alpha=1 / 201 / \mathrm{s}^{2}$ and $\beta=1 / 1.51 / \mathrm{s}$; with these values, cruise control systems are configured in a way that is perceived by drivers as agreeable and natural [14]. For the preferred gap $g^{*}(v)=v \tau$, as a rule the legal regulation is applied, albeit with a somewhat smaller value for the preferred time gap $\tau$, e.g. $\tau=1.5 \mathrm{~s}$, which is also used in the rest of this chapter. The model in Eq. (15.2) was originally introduced in Helly 1959 [15] as a model describing a human driver. This underscores the assertion that many driver models and the models for autonomous driving are mathematically very similar. Where they differ will be discussed in greater detail in Sect. 15.3.

The model in Eq. (15.2) has limits. For example, it is crash-free only for particular parameters $(\alpha, \beta)$, and is only string stable for a small subset of parameters. String stability is the ability of a chain of vehicles driving behind each other not to succumb to the "slinky effect" and jam up: for instance, when minor braking by the first vehicle in the chain leads to an amplified effect along the chain, in extreme cases actually causing a vehicle in the chain to come to a standstill. Or causing a traffic accident. To date, this behavior has only been found in very specific situations (see [21] for an example) - it does not appear to be the normal case.

However, the parameters with string stability are not perceived as very agreeable by human drivers, so AIC systems generally apply a compromise solution that results in a weak string instability [14].

For that reason, this paper looks at a different approach in the tradition of the models in [16-18]. A first step considers that an important condition for safe driving is fulfilled when the following applies:

$$
d(v)+v \tau \leq D(V)+g .
$$

In this equation, $D(V), d(v)$ are the braking distances of the leading and following vehicles. Obviously this model is predicated on the following driver having an idea of whether and how the leading vehicle will drive or brake. That is certainly not entirely adequate; and yet driving does work in many cases on the assumption that the other drivers will behave more or less as one does oneself. 
However, that also means that the approach flowing from this and the following equation can be tricked by "strange" behavior on the part of the leading vehicle. If the leading vehicle has an autonomous emergency braking system that allows deceleration values of up to $12 \mathrm{~m} / \mathrm{s}^{2}$, it violates the assumption of similar behavior to the following vehicle - typical deceleration values for a human driver are in the range of up to max. $4 \mathrm{~m} / \mathrm{s}^{2}$ - leading to a much shorter braking distance. This can be compensated for to some extent, as the following simulation results also show, because the equations resulting from this approach in the case of strong braking by the leading vehicle can exceed their own deceleration. At the same time, this approach is one that could find further application in the development of driver models for traffic safety.

The above model can be developed further by stipulating that the safety condition be fulfilled not at the current time $t$, but also for a certain time $t+T$ in the future. The time $T$ is the anticipation time, i.e. the length of the planning horizon of the driver. With the notation $x^{\prime}$ as a short-hand for the value of the variable $x$ at the time $t+T$, the safety equation becomes:

$$
d\left(v^{\prime}\right)+v^{\prime} \tau \leq D\left(V^{\prime}\right)+g^{\prime}
$$

But this equation can now be reformulated according to acceleration $a$. Thus $x^{\prime}=$ $x+v T+a T^{2} / 2$ and together with an approach for the braking distances $d(v)=v^{2} /(2 b)$, the safety equation can be solved for $a$. There are various approaches for this; here primarily the following exact approach is pursued:

$$
\dot{v}=\frac{1}{T}\left(-b(\tau+T / 2)+\sqrt{b^{2}(\tau+T)^{2}+V^{2}+2 b v T+2 b(g+\Delta v T)}-v\right) .
$$

Interestingly, this approach for $T \rightarrow 0$ leads back to the one used in SUMO [19].

Another possibility, following [17], is a Taylor expansion of $d\left(v^{\prime}\right)=d(v+a T) \approx d(v)+a T v / b(v)$, which, interestingly, leads to a linear equation for $a$ which is simpler to solve and numerically less complex:

$$
a=\frac{V^{2}-v^{2}+2 b(T \Delta v+g-v \tau)}{T(2 b \tau+b T+2 v)} .
$$

Although these equations look complicated, and it is rather unlikely that people can actually extract a root from a complex expression while driving, graphically it does strongly resemble the Helly model. This is interesting because it is indeed quite easy to imagine that a human driver is capable of carrying out a linear consideration along the lines of "I'm moving somewhat faster than the person in front of me, but the gap is large, so there is no immediate need to change anything." An idea of how this acceleration function looks for realistically selected parameters is provided by Fig. 15.2. 

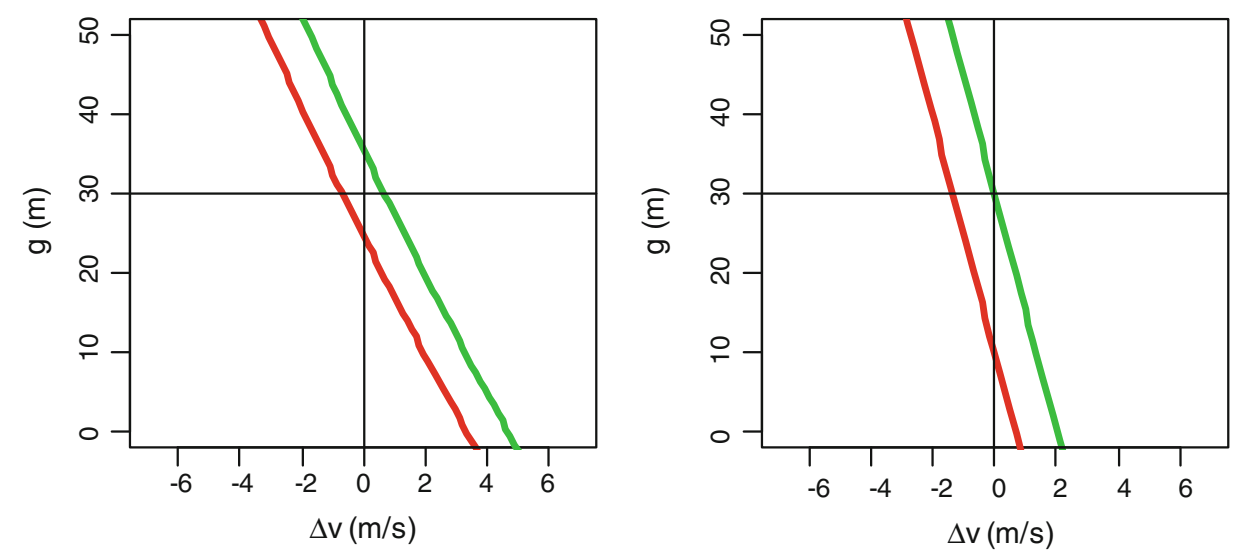

Fig. 15.2 Representation of the acceleration functions. Rather than drawing the entire function here, only the area delimited by two lines in the $(\Delta v, g)$ range is represented, in which the acceleration of both models is small. To the left of the lines, the vehicle is braking, to the right it is accelerating. The figure on the left is the model from Eq. (15.3), the one on the right the Helly model (15.2). The selected parameters are $V=20, \tau=1.5, b=4, T=2$. Image rights: copyright resides with author

In this context it is also interesting to know whether this approach is indeed free of collisions. The simple answer is no. Under some circumstances, the dynamic that follows from Eq. (15.3) can indeed be fooled. This can be demonstrated by a chain of vehicles following a leading vehicle that is driving according to a specific protocol $a_{0}(t)$. The salient parameters in the dynamics of the leading vehicle are primarily the maximum accelerations. Of particular interest here are the maximum decelerations and the question as to whether it is possible to produce a collision with the model.

Of course, no procedure can really test all eventualities. But the following approach does at least allow an estimation of how secure the models are. In a simulation, $n=50$ vehicles follow a leading vehicle that selects its acceleration according to a specific protocol. Among other things, it repeatedly decelerates to a standstill, in some cases with decelerations at the limits of current driving dynamics capabilities. Studies on this set-up very quickly revealed that collisions can only be avoided in the models when the anticipation time $T$ during braking is set to a lower value. In the following the models are always operated with $T=2 \mathrm{~s}$ in normal driving, and with $T=0.5 \mathrm{~s}$ when braking.

The respective simulations then show that, under these conditions, no accidents occur with the model in Eq. (15.3), at least not with the selected protocol $a_{0}(t)$. The Helly model, however, is not so tolerant with the selected parameters and occasionally produces rear-end collisions. 


\subsection{Man Versus Machine}

At this point the question arises as to what actually distinguishes a human driver from an autonomous vehicle. Heretofore just one significant difference has been established, and that is the time gap $\tau$ with which the two drive. Humans should not drive with a gap of less than $\tau=0.9 \mathrm{~s}$, and the legal recommendation is actually $\tau=2 \mathrm{~s}$; in principle, a machine can drive with a $\tau=0.3 \ldots 0.5 \mathrm{~s}$ gap [1]. An example analysis of the actually maintained gaps (see Fig. 15.3) on a German autobahn (with speed around $100 \mathrm{~km} / \mathrm{h}$, where the greatest traffic volumes are achieved) shows that (very) few human drivers approach this "ideal", whereas the overwhelming majority demonstrates legally compliant behavior.

Figure 15.3 also shows that human behavior covers a broad spectrum which stands in contrast to autonomous vehicles: they would all drive with a small and very similar value of $\tau$. This spectrum can be characterized [20] and quantified in greater detail. In general it can be said that $\tau$ not only varies between different drivers, but indeed is not even consistent for the same driver. Unfortunately $\tau$ is not precisely observable, in particular when the leading vehicle itself constantly changes speed, so it is only possible to posit assumptions as to how $\tau$ varies over time. This then leads to 2D models [20, 21], in which $\tau$ varies in each time step. A simple scenario that leads to such a dynamic is the driver misjudging the gap. However, this error is time-correlated, i.e. when the estimated gap at a certain point in time is smaller than the actual gap, this will continue to be the case for a certain period thereafter as well. And there is a reasonable probability that the error will be asymmetrical: Gaps are frequently estimated to be significantly smaller than they actually are. Such a modeling approach does in any case lead to a very broad spectrum of $\tau$ values, just as is observed empirically.

A second point in which a human differs from a machine is what is known as the action point mechanism [22]. Strictly speaking, a human driver cannot be described by a differential Eq. (15.1). Rather, a vehicle is controlled through correction of the acceleration (accelerator-pedal position) at irregular time intervals, as shown in the example Fig. 15.4.

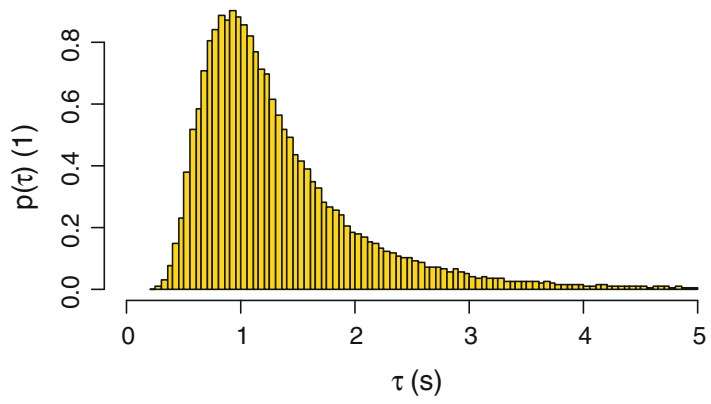

Fig. 15.3 Gap behavior in the left lane of the A3. Displayed is the density of the respective time gap. The maximum of the function is more or less precisely $1.1 \mathrm{~s}$, while the average is $1.4 \mathrm{~s}$. Some dangerously short time gaps are observed, too. Image rights: copyright resides with author 


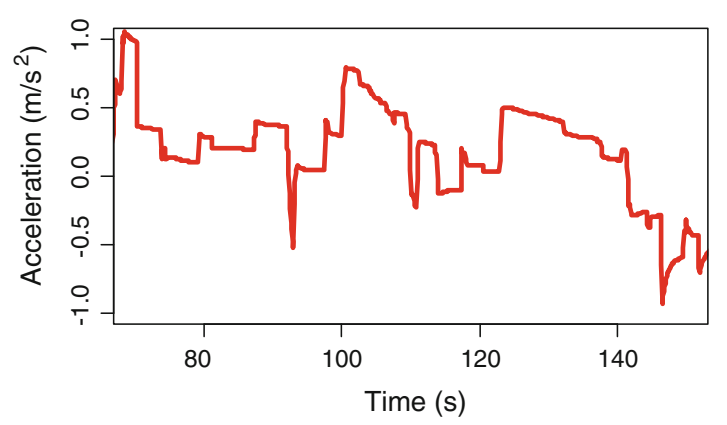

Fig. 15.4 Acceleration as a function of time with a human driver. It can be seen that the acceleration changes erratically at the action points. Between the action points, it remains nearly constant. The data was recorded in a "drive" by the author with a driving simulator; similar images can be found in all data records with sufficiently accurate measurement of the acceleration or accelerator and brake pedal. Image rights: copyright resides with author

The time gaps between successive action points also demonstrate a very broad distribution, with values between 0.5 and $1.5 \mathrm{~s}$. Here there is evidently another modeling approach for traffic safety questions - if the time between two action points becomes very long, a critical situation can arise. In normal cases that does not occur, however, and there are only minor variances between a model based on Eq. (15.1) and a model in which the action points are explicitly used [23]. In particular, the action point mechanism alone does not lead to a wide distribution of gaps between the vehicles.

This too is demonstrated in the example used in Sect. 15.2 of the chain of vehicles following a leading vehicle. An evaluation of the gap measured (in the simulation), here as a function of the number of the following vehicle, shows that in most cases an autonomous vehicle follows the leading vehicle with significantly less variance - in spite of the sometimes extremely volatile behavior. A representation of this is found in Fig. 15.5.

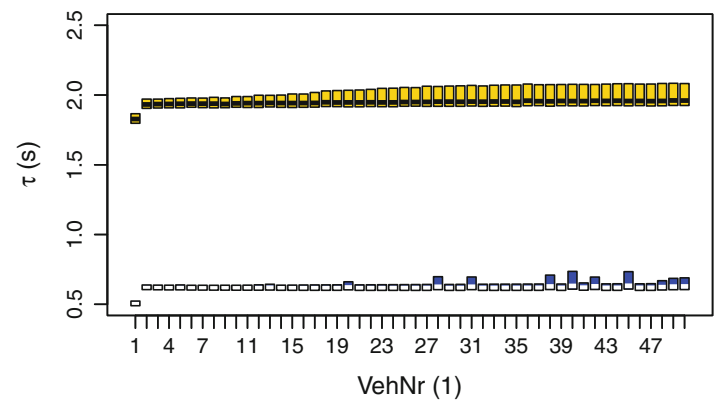

Fig. 15.5 Gap size behavior for human and autonomous vehicles. The graphic shows the average gap and the 25th and 75th percentiles, in each case as a function of the position in the chain. The upper curve is for the model of the human driver and the lower one models a chain of autonomous vehicles. Image rights: copyright resides with author 
Thus the models used in this chapter have been specified, and the difference between the human and the autonomous driving style has been characterized. The rest of this chapter will utilize various applications to illustrate what that means for typical traffic management applications.

\subsection{Approaching a Traffic Signal}

This process is one of the candidates in which autonomous vehicles promise significant benefits. In an approach to a traffic signal, the following examines the delay $d$ per vehicle for a random combination of normal and autonomous vehicles. Here, $\eta$ describes the share of autonomously driving vehicles, whereas $\tau=0.5 \mathrm{~s}$ is assumed for autonomous and $\tau=1.5 \mathrm{~s}$ for normal vehicles. The simulation results are also supported by a theoretical consideration. There is a theory for the described situation which was developed in [24]. Interestingly, the theory can be applied to a situation with a mix of autonomous and normal vehicles. Then the respective expression is:

$$
d(q, \eta)=\frac{c}{2} \frac{(1-\lambda)^{2}}{1-y}+\frac{1}{2} \frac{x^{2}}{q(1-x)}, \lambda=\frac{g}{c}, y=\frac{q}{s}, x=\frac{y}{\lambda}, s=s_{0}(1-\eta)+s_{1} \eta
$$

In Eq. (15.4), $q$ is the demand, $s_{0}$ the capacity of a flow of human-guided vehicles, $s_{1}$ the capacity of a flow of automated vehicles, $g$ the green time and $c$ the cycle time of the traffic signal. The cycle time is the time it takes for the traffic signal to regain the same state it had at the beginning. The simulation results for selected variations in demand $(q)$ and the share of autonomous vehicles $\eta$ is shown in Fig. 15.6.

The curves in Fig. 15.6 were recorded by simulating various values of demand $q$ (varying from 18 to $1800 \mathrm{veh} . / \mathrm{h}$ ) for $5 \mathrm{~h}$ each. The demand itself is a stochastic variable

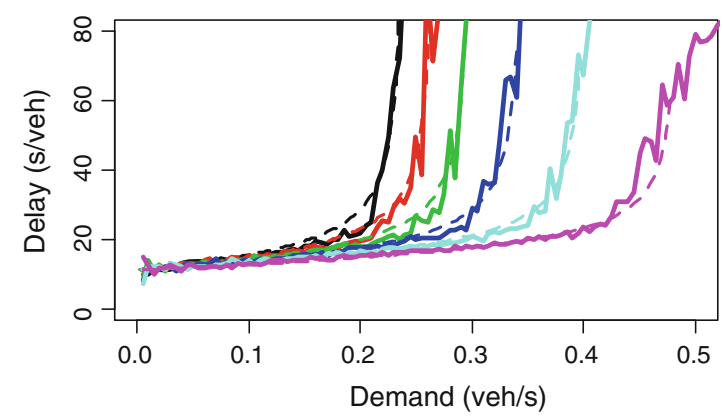

Fig. 15.6 Delay at a traffic signal as a function of the demand and for different equipment rates $\eta=0,20,40,60,80$ and $100 \%$ (from left to right). The dotted lines were calculated from Eq. (15.4), albeit with a capacity, which was measured directly in the simulation. Image rights: copyright resides with author 
(approximately Poisson-distributed), i.e. in each observed time interval, there is always a different number of vehicles and only the average over many such time intervals leads to the correct demand.

The delay was recorded for each simulated vehicle and the values used to calculate the average entered in Fig. 15.6. In principle, the entire distribution of delays can be used to characterize the results, which for reasons of space is omitted here, although it would be interesting. The fluctuations in the delays are a measure of the reliability of such a system. However, the example presented here shows that the delay fluctuations are only very weakly correlated with the proportion of autonomously guided vehicles; the major source of stochasticity in this system is generated by the demand and not the dynamics of the vehicles.

Two results in Fig. 15.6 stand out. For one thing, the description from the theory does not always correspond to the simulation results. A considerable amount of research is still needed here, because it's not at all simple to translate the assumptions on which the theory is based into the simulated reality. This will undoubtedly be even more difficult in comparison with real measured values. To achieve agreement, the values for the saturation-traffic volume determined in the simulation had to be used-with the theoretical values, i.e. the $\tau$ values defined in Sect. 15.3, the agreement is not compelling.

Second, autonomous vehicles "only" change the capacity; otherwise there are no or only very small gains. As long as the demand stays away from the respective capacity, there are only minor differences between the various scenarios, at least on the level of the description selected here.

A change in the capacity does have one very positive effect, however: it means that the required green times at a traffic signal can be shorter, leaving more time for other modes of transport.

\subsection{Adaptive Traffic Signals}

Section 15.4 looked at a traffic signal with a fixed-time control system. Many modern systems, however, utilize an adaptive control system. That means that the traffic signal attempts to coordinate its green times with the current demand. When demand is low, the

Fig. 15.7 Green times (left) and delays (right) in a simulated adaptive system, displayed as a function of the time and for different proportions of autonomous vehicles $\eta=0,25,50,75$, $100 \%$. The demand parameter was set to $q_{0}=180 \mathrm{veh} . / \mathrm{h}$ and $q_{1}=720$ veh./h. Image rights: copyright resides with author
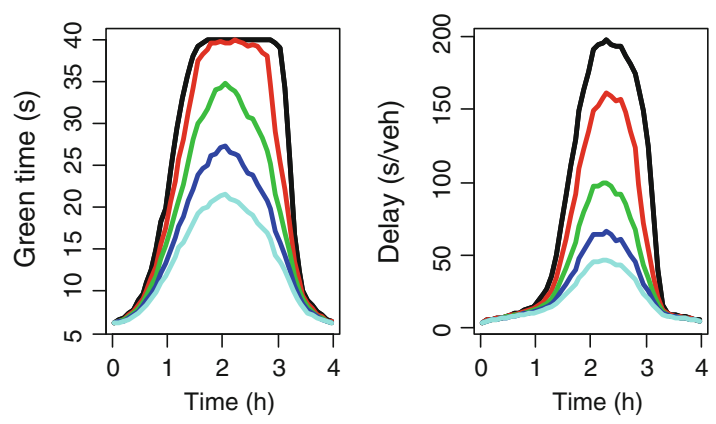
green times are short, and when demand is high, the system responds with long green times. The details are somewhat more complex, because the delay regarded as a function of the demand has a minimum with a certain optimal cycle time. An adaptive system is able to choose the optimal cycle time for itself, and makes very clever use of the fluctuations that occur in the traffic flow.

In this case as well, the aim is to examine how such an adaptive system handles a mix of autonomous and normal vehicles. To this purpose, simulation of a two-armed intersection controlled with an adaptive method was set up [27]. The two arms are $600 \mathrm{~m}$ long, and the delay per vehicle at intersection is measured. In contrast to Sect. 15.4, however, a demand was selected that depends on the time and thus replicates a peak hour group in which at the time of maximum demand the system is saturated in spite of its adaptivity. The demand function selected here is:

$$
q(t)=q_{0}+q_{1} \sin \left(\frac{\pi t}{T}\right)
$$

where $q_{0}$ is a basic load, $q_{1}$ is the amplitude of the demand fluctuation and $T$ is the entire time period of the simulation. Both arms are subjected to the same demand, which represents a relatively unfavorable case.

Beyond the delays, in this case it is primarily the green times that are of interest. Since the system adapts the times to the demand, they fluctuate within typical ranges. In many countries, the green time cannot fluctuate freely: for instance, the green time for a normal traffic signal cannot sink below $5 \mathrm{~s}$, and in the following simulations, the maximum green time is set to $40 \mathrm{~s}$.

Such a simulation is also an interesting case in the evaluation of the simulation data. A single simulation of such a peak hour shows major fluctuations in terms of delays as well as green time and cycle times. Although the delays were averaged over a cycle of the system, that in itself is not sufficient because the cycles are themselves stochastic variables

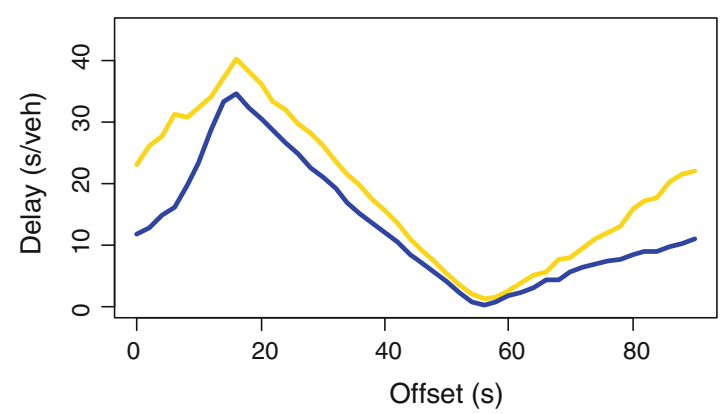

Fig. 15.8 Delay as a function of the offset time for a simple green wave. Depicted here is a simulation with human drivers (gold) and one only with autonomous vehicles (blue). Shown here is the best result achieved between the first and second intersection. Image rights: copyright resides with author 
whose average values and statistics can only be determined through a sufficient number of repetitions of the same scenario with slightly different details - just as in reality when successive days are examined. To obtain statistically valid results, in this case the peak hour was repeated 50 times. At 5-min intervals, the averages of the delays over the last cycle and the corresponding green times set by the system were collected. The results in Fig. 15.7 were composed from this data.

With maximum demand, the system extends the green times up to the limit of $40 \mathrm{~s}$ and thereby demonstrates that it has reached its saturation level. However, this only applies for a flow of normal vehicles. As soon as autonomous vehicles are added to the mix, the top delay value for sinks and with an equipment rate of $50 \%$, the maximum green time is not even reached. This lines up with the observation in Sect. 15.4 that autonomous vehicles not only increase the capacity, but also contribute to a reduction in green times - an effect that is rather clear in this example, namely that even a small proportion of autonomously guided vehicles can make a noticeable impact.

\subsection{Green Wave with Autonomous Vehicles}

The previous scenarios examined a single intersection. Much more interesting is the case of a stretch of road with multiple intersections in succession which are all controlled by a traffic signal system. In this case the coordination between the traffic signals, known

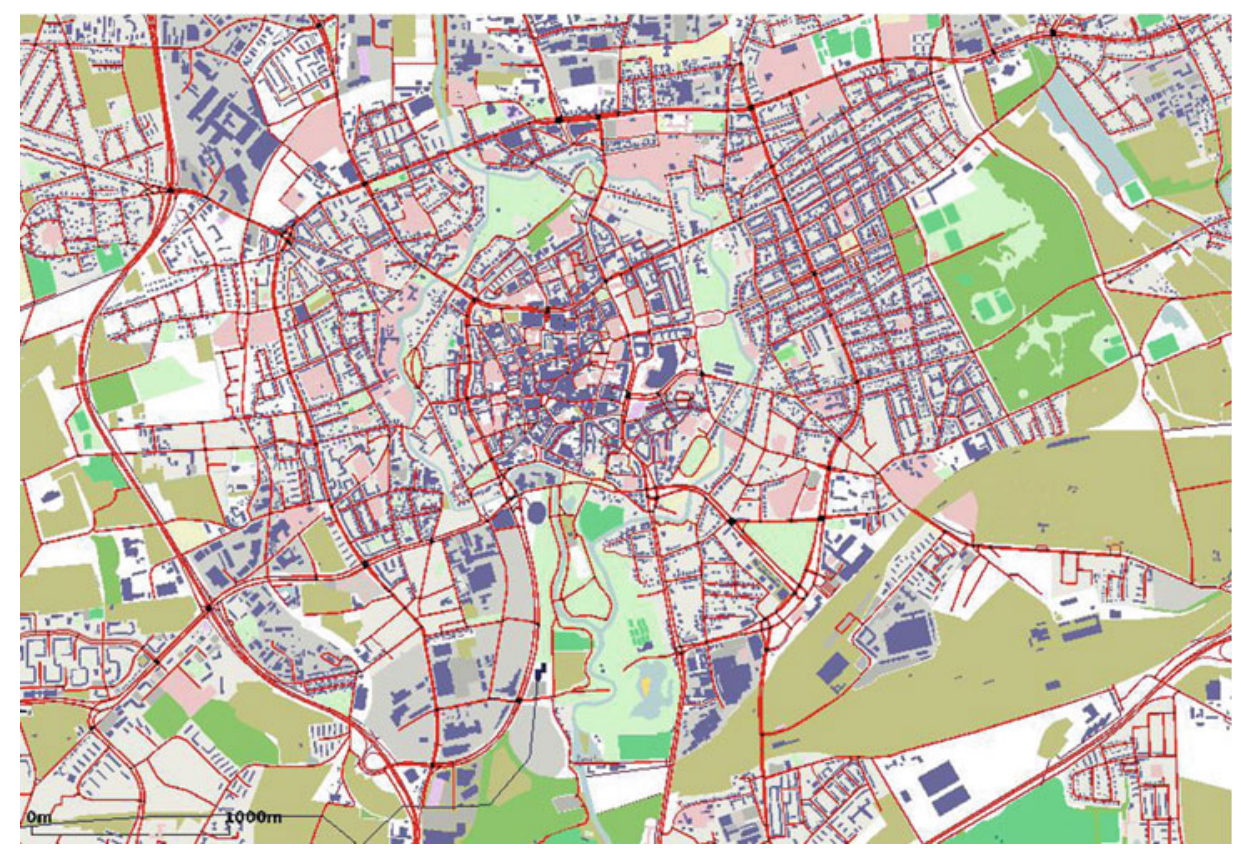

Fig. 15.9 Excerpt of the simulation network for Braunschweig. The land use data comes from the openstreetmap dataset [26]. Image rights: copyright resides with author 
colloquially as the green wave, plays an important role. Here again, a simulation is used to investigate how great an impact the introduction of autonomous vehicles has. Analogous to the procedure in [28], a section of road with 10 intersections is simulated with varying coordination configurations. The demand, which is constant, the green times and the cycle times remain unchanged. The only change is to the offset, i.e. the point in time at which the traffic signal turns green for the vehicle flow in a particular direction. If this offset between two signals is precisely equal to the travel time between the two signals, the system is in its optimal state: the delay for the vehicles at the downstream signal is exactly zero when the green times are equal. In that case, just as many vehicles can cross the intersection as left the upstream traffic signal.

The expectation is clear: In this case, no improvements will be achieved with an autonomous vehicle; and that is precisely what the simulation results in Fig. 15.8 demonstrate. However, autonomous vehicles do indeed improve the delay times in the case of sub-optimal coordination. The reason is that the bunch of vehicles that leaves a traffic signal is more compressed than with human drivers.

\subsection{Simulation of a City}

This final section will examine how the introduction of autonomous vehicles might impact an entire city. To this purpose, an existing SUMO simulation [19 25] of the city of Braunschweig is used to simulate the impact of autonomous vehicles on the traffic flow of a transportation system.

However, the model introduced in Sect. 15.2 is not implemented in SUMO, so the simulation has to be carried out with the models that are available in SUMO. The simulation therefore uses the standard model integrated in SUMO, which in terms of describing the fluctuations of the drivers is not as refined as the model introduced here.

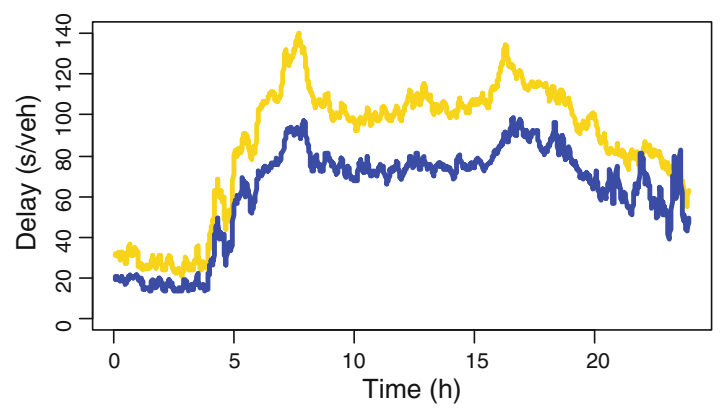

Fig. 15.10 Comparison of the delays for a simulation with human drivers (gold, upper curve) and a simulation in which the passenger vehicles drive autonomously (blue, lower curve). Each data point is a floating average value from the 8 adjacent one-minute values. The dispersion of the values of the two curves is not very different and is therefore not displayed. Image rights: copyright resides with author 
To set up the model, a modified network by the NavTeq company is used; an extract of the transportation network is seen in Fig. 15.9. The full simulation comprises the entire area of the city of Braunschweig, including the autobahns in the area. The simulation network comprises approximately 129,000 edges.

The required traffic demand comes from a start/destination matrix from the PTV company, which is available for different days of the week in 24 time slices of one hour each for each of those days. This demand was used to calculate a user equilibrium, which in this case required some 100 iteration steps. At the end of this process, for each vehicle simulated in SUMO there is an optimal route in the sense that every other route through the network would take longer. A total of 647,000 vehicles were simulated. Initial comparisons with real data from Braunschweig suggest that the matrix significantly underestimates the demand. This undoubtedly affects the results discussed here, but it was not possible to carry out such corrections in the context of this project.

To simulate autonomous vehicles, a new vehicle type is introduced which has similar parameters to the models in Sect. 15.2: the autonomous vehicles in SUMO drive with $\tau=0.5 \mathrm{~s}$, all others with $\tau=1$ and $\sigma=0.5 . \sigma$ is the noise parameter in SUMO, i.e. it indicates by how much a vehicle deviates from the optimal driving style. The selection of $\tau=0.5 \mathrm{~s}$ means that the time-step size in SUMO also has to be set to $0.5 \mathrm{~s}$ to ensure that the vehicles can continue to drive without colliding. This extends the simulation time from around $50 \mathrm{~min}$ to $90 \mathrm{~min}$ for the simulation of an entire day in Braunschweig.

Only the passenger vehicles were simulated as autonomous vehicles; the approximately 44,000 trucks remained unchanged. The traffic signals were likewise not entirely correctly represented in the simulation. It may therefore be assumed that on this end as well, further corrections to the simulation results below can be expected.

Nevertheless, this simulation delivers significant preliminary results, as seen in Fig. 15.10. Even without further measures, the autonomous system is more efficient in the sense that it reduces delays between 5 and $80 \%$, with an average value of around $40 \%$. With the selected parameters, however, the variance in travel times changes relatively little; the system, in other words, becomes faster, but not necessarily more reliable. That could change if the traffic management system were also realistically simulated. Such studies are currently in the works.

\subsection{Conclusion}

This paper presents some initial considerations regarding how traffic management needs to respond to the opportunities presented by autonomous driving. The case studies presented here demonstrate that, depending on the scenario, very different improvements can be achieved in the flow of traffic through the introduction of autonomous vehicles.

Unfortunately, the improvements that could be achieved are difficult to summarize with a single number. It was demonstrated in Sect. 15.4, for example, that the capacity of a traffic signal can certainly be doubled. If the demand is low at the corresponding signal, 
this doubling is scarcely noticeable. But if the signal is working at the limits of its capacity, by contrast, even a minor increase in its capacity can lead to a dramatic improvement.

This can be observed quite clearly in the scenario in Sect. 15.5: here the demand runs the values from very low to (temporary) over-saturation. Although the introduction of autonomous vehicles has little impact on green times and delays when demand is low, it yields major improvements when the system is operating beyond capacity. Nevertheless, the magnitude of these improvements does depend on the details of the scenario being examined. If the peak value for demand were just a bit lower, the benefit would also be significantly diminished.

That notwithstanding, it may be asserted with confidence that at least in the urban context, the introduction of autonomous vehicles has the potential to generate substantial time gains at traffic signals which would then be available for other road users - if the introduction of these vehicles does not lead to an increase in demand for automotive transportation.

Open Access This chapter is distributed under the terms of the Creative Commons Attribution 4.0 International License (http://creativecommons.org/licenses/by/4.0/), which permits use, duplication, adaptation, distribution and reproduction in any medium or format, as long as you give appropriate credit to the original author(s) and the source, a link is provided to the Creative Commons license and any changes made are indicated.

The images or other third party material in this chapter are included in the work's Creative Commons license, unless indicated otherwise in the credit line; if such material is not included in the work's Creative Commons license and the respective action is not permitted by statutory regulation, users will need to obtain permission from the license holder to duplicate, adapt or reproduce the material.

\section{References}

1. Winner, H.: private correspondence. (2014)

2. Friedrich, B.: The Effect of Autonomous Vehicles on Traffic. Present volume (2014)

3. Pavone, M.: The Value of Robotic Mobility-on-Demand Systems. Present volume (2014)

4. van Dijke, J., van Schijndel, M., Nashashibi, F., de la Fortelle, A.: Certification of Automated Transport Systems. Procedia - Social and Behavioral Sciences 48, 3461 - 3470 (2012)

5. Kesting, A.: Microscopic Modeling of Human and Automated Driving: Towards Traffic-Adaptive Cruise Control, Verlag Dr. Müller, Saarbrücken, ISBN 978-3-639-05859-8 (2008)

6. Reuschel, A.: Fahrzeugbewegung in der Kolonne bei gleichförmig beschleunigtem oder verzögertem Leitfahrzeug. Zeitschrift des österreichischen Ingenieur und Architektenvereins, 7/8, 95 - 98 (1950)

7. Chowdhury, D., Santen, L., Schadschneider, A.: Statistical physics of vehicular traffic and some related systems. Physics Reports 329, 199 - 329 (2000)

8. Helbing, D.: Traffic and Related Self-Driven Many-Particle Systems. Reviews of Modern Physics 73, 1067 - 1141 (2001)

9. Nagel, K., Wagner, P., Woesler, R.: Still flowing: approaches to traffic flow and traffic jam modelling. Operations Research 51, 681 - 710 (2003)

10. Treiber, M., Kesting, A.: Traffic Flow Dynamics: Data, Models and Simulation. (2012) 
11. Urmson C., et al: Autonomous Driving in Urban Environments: Boss and the Urban Challenge. Journal of Field Robotics 25, 425 - 466 (2008)

12. Levinson, J. et al.: Towards fully autonomous driving: Systems and algorithms. In proceedings of the 2011 IEEE Intelligent Vehicles Symposium, 163 - 168 (2011)

13. Campbell M., Egerstedt, M., How, J. P., Murray, R. M.: Autonomous driving in urban environments: approaches, lessons and challenges. Philosophical Transactions of the Royal Society A 368, 4649 - 4672 (2010)

14. Winner, H., Hakuli, S., Wolf, G.: Handbuch Fahrerassistenzsysteme: Grundlagen, Komponenten und Systeme für aktive Sicherheit und Komfort (2011)

15. Helly, W.: Simulation of bottlenecks in single lane traffic flow. Proceedings of the symposium on theory of traffic flow (1959)

16. Gipps, P.: A behavioural car-following model for computer simulation. Transportation Research Part B 15, 105 - 111 (1981)

17. Krauß, S.: Microscopic modelling of traffic flow: Investigation of Collision Free Vehicle Dynamics, Dissertation, Universität zu Köln (1998)

18. Krauß, S., Wagner, P., Gawron, C.: Metastable states in a microscopic model of traffic flow. Physical Review E 55, 5597 - 5602 (1997)

19. Krajzewicz, D, Erdmann, J., Behrisch, M, Bieker, L.: Recent Development and Applications of SUMO - Simulation of Urban MObility. International Journal On Advances in Systems and Measurements, 5, 128 - 138 (2012)

20. Wagner, P.: Analyzing fluctuations in car-following. Transportation Research Part B 46, 1384 $1392(2012)$

21. Jiang, R., Hu, M., Zhang, H.M., Gao, Z., Jia, B., Wu, Q., Wang, B., Yang, M.: Traffic Experiment Reveals the Nature of Car-Following. PLoS ONE 9: e94351. doi:10.1371/journal. pone.0094351 (2014)

22. Todosiev, E.P., L. C. Barbosa, L.C.: A proposed model for the driver-vehicle-system. Traffic Engineering, 34, 17 - 20, (1963/64)

23. Wagner, P.: A time-discrete harmonic oscillator model of human car-following. European Physical Journal B 84, 713 - 718 (2011)

24. Webster, F.V.: Traffic Signal Settings. Department of Scientific And Industrial Research Road Research Laboratory, (1958)

25. Krajzewicz, D., Furian, N., Tomàs Vergés, J.: Großflächige Simulation von Verkehrsmanagementansätzen zur Reduktion von Schadstoffemissionen. 24. Verkehrswissenschaftliche Tage Dresden, Deutschland (2014)

26. OpenStreetMap: www.openstreetmap.org, last accessed 7/29/2014

27. Oertel, R., Wagner, P.: Delay-Time Actuated Traffic Signal Control for an Isolated Intersection. In: Proceedings 90th Annual Meeting Transportation Research Board (TRB) (2011)

28. Gartner, N.H., Wagner, P.: Traffic flow characteristics on signalized arterials. Transportation Research Records 1883, 94 - 100 (2004) 